\title{
Mental Health Among Otolaryngology Resident and Attending Physicians During the COVID-19 Pandemic: A National Study
}

\author{
Karthik Rajasekaran, $\mathrm{MD}^{1}$ \\ ${ }^{1}$ University of Pennsylvania
}

May 12, 2020

\begin{abstract}
Background: Otolaryngologists are amongst the highest risk for COVID-19 exposure.

Methods: A cross-sectional, survey-based, national study evaluating academic otolaryngologists. Burnout, anxiety, distress, and depression were assessed by the single-item Mini-Z burnout assessment, 7-item Generalized Anxiety Disorder scale, 15-item Impact of Event Scale, and 2-item Patient Health Questionnaire, respectively.

Results: 349 physicians completed the survey. 165 (47.3\%) were residents and 212 (60.7\%) were males. Anxiety, distress, burnout and depression were reported in $167(47.9 \%), 210(60.2 \%), 76(21.8 \%)$ and $37(10.6 \%)$ physicians respectively. Attendings had decreased burnout relative to residents (OR 0.28, CI [0.11-0.68]; $\mathrm{p}=0.005$ ). Females had increased burnout (OR 1.93, CI [1.12.3.32 ]; $\mathrm{p}=0.018$ ), anxiety (OR 2.53, CI [1.59-4.02]; $\mathrm{p}<0.005)$, and distress (OR 2.68, CI [1.64-4.37]; $\mathrm{p}<0.005$ ). Physicians in states with greater than 20,000 positive cases had increased distress (OR 2.01, CI [1.22-3.31]; $\mathrm{p}=0.006$ ).

Conclusion: During the COVID-19 pandemic, the prevalence of burnout, anxiety, and distress is high among academic otolaryngologists.
\end{abstract}

Alyssa M. Civantos, BA, ${ }^{1}$ Yasmeen Byrnes, BA, ${ }^{1}$ Changgee Chang, $\mathrm{PhD},{ }^{2}$ Aman Prasad, BS, ${ }^{1}$ Kevin Chorath, BS, ${ }^{3}$ Seerat K. Poonia, MD, ${ }^{4}$ Carolyn M. Jenks, MD ${ }^{4}$ Andrés M. Bur, MD ${ }^{5}$ Punam Thakkar, MD ${ }^{6}$ Evan M. Graboyes, MD, MPH,${ }^{7}$ Rahul Seth, MD, ${ }^{8}$ Samuel Trosman, MD,${ }^{9}$ Anni Wong, MD,${ }^{9}$ Benjamin M. Laitman, MD, PhD,${ }^{9}$ Brianna N. Harris, MD, ${ }^{10}$ Janki Shah, MD,${ }^{11}$ Vanessa Stubbs, MD,${ }^{12}$ Garret Choby, $\mathrm{MD},{ }^{13}$ Qi Long, $\mathrm{PhD},{ }^{2}$ Christopher Rassekh, MD ${ }^{4}$ Erica Thaler, $\mathrm{MD},{ }^{4}$ Karthik Rajasekaran, $\mathrm{MD}^{4}$

${ }^{1}$ Perelman School of Medicine, University of Pennsylvania, Philadelphia, PA

${ }^{2}$ Department of Biostatistics, Epidemiology and Informatics, University of Pennsylvania, Philadelphia, PA

${ }^{3}$ Long School of Medicine, University of Texas Health San Antonio, San Antonio, TX

${ }^{4}$ Department of Otorhinolaryngology, University of Pennsylvania, Philadelphia, PA

${ }^{5}$ Department of Otolaryngology, University of Kansas School of Medicine, Kansas City, KS

${ }^{6}$ Department of Otolaryngology, George Washington University, Washington, D.C

${ }^{7}$ Department of Otolaryngology, Medical University of South Carolina, Charleston, SC

${ }^{8}$ Department of Otolaryngology, University of San Francisco, San Francisco, CA

${ }^{9}$ Department of Otolaryngology, Mt. Sinai Health System, New York City, NY

${ }^{10}$ Department of Otolaryngology, Scripps Health/Senta Clinic, San Diego, CA

11 Department of Otolaryngology, Cleveland Clinic, Cleveland, $\mathrm{OH}$

12 Department of Otolaryngology, University of Miami, Miami, FL 
${ }^{13}$ Department of Otolaryngology, Mayo Clinic, Rochester, MN

Address correspondence and reprint request to:

Karthik Rajasekaran, MD,

Department of Otorhinolaryngology-Head and Neck Surgery,

800 Walnut St, $18^{\text {th }}$ Floor,

Philadelphia, PA 19107

Email: Karthik.Rajasekaran@pennmedicine.upenn.edu

Disclosures: This work has never been published or presented anywhere. The authors have no financial or industry relationships to disclose.

Funding: This work was supported by the Nationtal Institute of Health P30 CA016520 grant (QL).

Running title: Mental Health among otolaryngologists during COVID-19

Keywords: COVID-19, mental health, mental wellness, otolaryngologists, psychiatric distress

\begin{abstract}
Background: Otolaryngologists are amongst the highest risk for COVID-19 exposure.

Methods: A cross-sectional, survey-based, national study evaluating academic otolaryngologists. Burnout, anxiety, distress, and depression were assessed by the single-item Mini-Z burnout assessment, 7-item Generalized Anxiety Disorder scale, 15-item Impact of Event Scale, and 2-item Patient Health Questionnaire, respectively.
\end{abstract}

Results: 349 physicians completed the survey. 165 (47.3\%) were residents and $212(60.7 \%)$ were males. Anxiety, distress, burnout and depression were reported in 167 (47.9\%), $210(60.2 \%), 76(21.8 \%)$ and 37 $(10.6 \%)$ physicians respectively. Attendings had decreased burnout relative to residents (OR 0.28, CI [0.11$0.68] ; \mathrm{p}=0.005$ ). Females had increased burnout (OR 1.93, CI [1.12.-3.32]; $\mathrm{p}=0.018$ ), anxiety (OR 2.53, CI [1.59-4.02]; $\mathrm{p}<0.005$ ), and distress (OR 2.68, CI [1.64-4.37]; $\mathrm{p}<0.005)$. Physicians in states with greater than 20,000 positive cases had increased distress (OR 2.01, CI [1.22-3.31]; $\mathrm{p}=0.006$ ).

Conclusion: During the COVID-19 pandemic, the prevalence of burnout, anxiety, and distress is high among academic otolaryngologists.

\title{
Introduction
}

The COVID-19 pandemic has presented healthcare providers with a unique set of challenges. While all healthcare providers have some exposure risk, it is particularly pronounced in those caring for patients undergoing mucosal or aerosol-generating procedures. ${ }^{1-3}$ At baseline, there are increased risks of acute respiratory infections among health care workers during procedures such as tracheal intubation, tracheotomy, non-invasive ventilation, and manual ventilation. ${ }^{4}$ Anecdotal reports from Wuhan, China report higher rates of COVID-19 infection specifically among otolaryngologists, likely due to the frequent use of those high-risk procedures in their typical, often non-elective, practice. ${ }^{5-9}$ This risk of exposure can be mitigated through the use of personal protective equipment (PPE); however, this essential resource is already becoming scarce. ${ }^{10}$ Additionally, they are being faced with increasing numbers of positive cases, unfamiliar hospital roles, and concern for 
safety of themselves, their loved ones, and their patients. Thus, beyond the risk to their physical health, there is also great risk to their mental wellbeing.

A review of prior outbreaks demonstrates a history of a mental burden on health care providers during similar times. A number of studies conducted during the 2003 SARS and 2014 Ebola crises used surveys and in-depth interviews to demonstrate increased symptoms of stress, anxiety, depression, insomnia, and distress among health care workers. ${ }^{11-17}$ In certain situations, investigators still found increased psychiatric morbidity one to three years after the 2003 SARS outbreak, though it is unclear whether these can be directly attributable to SARS. ${ }^{18-20}$ There are now similar concerns for the health care workers during the COVID-19 pandemic. Studies from COVID-19 in Wuhan, China, where it was first detected, as well as in Singapore and India have already shown an increased mental strain on healthcare workers, reflected through validated surveys on anxiety, depression, insomnia, and distress. ${ }^{21-23}$ This collection of prior evidence suggests a need to provide early support and intervention in the hopes of preventing any immediate or long-lasting implications.

Despite the history of increased psychiatric symptoms in health care workers during outbreaks and the increased infection risk among providers exposed to aerosolization, to the best of our knowledge, no study has characterized the impact on the mental health of this specific population. This study aims to assess mental health symptoms among Otolaryngology physicians during the COVID-19 pandemic by measuring symptoms of burnout, anxiety, depression, and distress.

\section{Methods}

This is a cross-sectional, survey-based, national study conducted during the COVID-19 pandemic from April 14, 2020 to April 25, 2020. The self-administered, anonymous online survey collected demographic data and mental health measurements from otolaryngology physicians from academic institutions throughout the United States. Participation was voluntary, and participants were allowed to terminate the survey at any time. A REDCap (Research Electronic Data Capture) database was developed specifically for this project and used to capture survey data. It was accessible only to study personnel. This project was reviewed and determined to qualify as quality improvement by the University of Pennsylvania's Institutional Review Board.

We contacted otolaryngology program directors via e-mail from all 109 allopathic academic programs in the US to disperse the survey to their residents, fellows, and attendings. Demographic data were self-reported by the participants, including sex (male or female), age, occupation (attending physicians, fellows, resident physicians), and geographic location. Date of projected peak resource utilization for each state was obtained from the Institute for Health Metrics and Evaluation'sCOVID-19 Projections in order to categorize participants based on the "Surge status" of their state. ${ }^{24}$ States reaching their date of projected peak resource use during our study period were in the "Surge", while states that had not reached that date were "Pre Surge," and states that were already past that date were "Post Surge." Numbers of positive COVID-19 cases and numbers of COVID-19 deaths per state were obtained from the COVID Tracking Projectfrom date April 19, 2020 , the midpoint of our study period. ${ }^{25}$

We focused on symptoms of burnout, anxiety, distress, and depression for all participants, using validated measurement tools. ${ }^{26-30}$ The single-item Mini-Z burnout assessment (range, 1-5) was used to assess burnout, with burnout defined as $>3{ }^{27,28}$ The 7-item Generalized Anxiety Disorder (GAD-7) scale (range, 0-21) was used to assess symptoms of anxiety over the past two weeks, with a scale of normal (0-4), mild (5-9), moderate (10-14), and severe (15-21) anxiety. ${ }^{26} \mathrm{~A}$ score of 10 has been reported to be a cut-off point for identifying cases of GAD. The GAD-7 included a final question assessing the "difficulty [these problems] made it for you to do your work, take care of things at home, or get along with other people" (range, 0-3). The 15-item Impact of Event Scale (IES; range, 0-75) was used to assess symptoms of distress over the past seven days, with a scale of subclinical (0-8), mild (9-25), moderate (26-43), and severe (44-75) distress. ${ }^{29}$ A score of 27 has been reported as a cut-off for risk of post-traumatic stress disorder (PTSD). ${ }^{31}$ The IES total score was also divided into two sub-scores: intrusion (range, 0-35) and avoidance (range, 0-40). Per Horowitz et al., the intrusion sub-scores assessed symptoms of "unbidden thoughts and images, troubled dreams, strong pangs or waves of 
feelings, and repetitive behavior." 29 The avoidance sub-score measured "ideational constriction, behavioral inhibition and counterphobic activity, and awareness of emotional numbness." ${ }^{29}$ The 2-item Patient Health Questionnaire (PHQ-2; range, 0-6), was used to assess symptoms of depression over the past two weeks, with a score of 3 as the cut-off for a positive depression screening requiring further evaluation with the more in-depth PHQ-9. ${ }^{30}$ These categories were based on values established in the literature. ${ }^{26-30}$

Data analysis was performed using $\mathrm{R}$ software version 3.6.3. The difference in distribution of symptoms across multiple groups is tested by the chi-square independence test (Table $\mathbf{2}$ ) and by the nonparametric Wilcoxon rank sum test and Kruskal-Wallis test (Table 3 ). To determine risk factors for severity of burnout, anxiety, distress, and depression, multiple logistic regression models were used (Table 4 ). The binary outcome variables were created for anxiety (normal vs other categories) and for distress (subclinical vs other categories). Type of physician, sex, age, surge status, and number of positive cases were included in the model, while location and number of deaths were found to be highly correlated with the number of positive cases and therefore excluded to alleviate the issue of collinearity. All tests were two-sided and the significance level $\alpha=0.05$ was applied. $95 \%$ confidence intervals were constructed, where applicable.

\section{Results}

\section{Baseline Characteristics}

A total of 349 physicians completed the survey. Of these, 165 [47.3\%] were residents, and 184 [52.7\%] were attending physicians, of which 12 were fellows. 1614 otolaryngology residents and 2849 otolaryngology fellows and attendings work at the academic institutions contacted, based on review of each institution's website. Thus, our response rate is $10.22 \%$ for residents and $6.46 \%$ for attendings, though these may be underestimates as we do not know whether all programs ultimately dispersed the survey to their physicians. Table 1 lists the demographic variables for the entire population. Most participants were men (212 [60.7\%]), and the most common age range was 31-35 years (114 [32.7\%]). Our male to female breakdown is consistent with that of the otolaryngology population, where $63.9 \%$ of residents and $85 \%$ of attendings are estimated to be males. ${ }^{32,33} 126(36.1 \%)$ participants worked in the Midwest, 107 (30.7\%) worked in the Northeast, 75 $(21.5 \%)$ in the South, and $41(11.7 \%)$ in the West. The majority came from states projected to reach their peak resource use during the study period (205[58.7\%]). 54.2\% of participants came from states estimated to have greater than 20,000 confirmed positive COVID-19 cases, and 54.2\% came from states estimated to have greater than 1,000 COVID-19 deaths.

\section{Mini-Z Burnout Scale Scores}

Burnout was reported in $76(21.8 \%)$ of participants. A greater distribution of resident physicians were experiencing burnout compared to attending physicians (49 [29.7\%] vs. 27 [14.7\%]; $\mathrm{p}=0.001$ ). A significantly greater proportion of female physicians also reported burnout compared to male physicians (40 [29.2\%] vs. $36[17.0 \%] ; \mathrm{p}=0.010$ ) (Table 2A ). The median (interquartile range, IQR) scores on the Mini-Z burnout measurement for all participants was 2.0 (2.0-2.0). As with the results grouped by the cutoff point, analysis of the median scores found residents to have a significantly increased score for burnout compared to attendings $(2.0$ [2.0-3.0] vs. $2.0[2.0-2.0] ; \mathrm{p}<0.0005)$, and females to have a significantly increased scores for burnout compared to males $(2.0$ [2.0-3.0] vs. $2.0[2.0-2.0] ; \mathrm{p}=0.004)$ (Table 3A ). Multivariable logistic regression analysis, which controlled for confounders, also showed that attendings were less likely to have positive screening for burnout compared to residents (OR 0.28, CI [0.11-0.68]; $\mathrm{p}=0.005$ ). Additionally, compared to male physicians, females physicians were more likely have a positive screening for burnout (OR 1.93, CI [1.12.-3.32]; $\mathrm{p}=0.018$ ) (Table 4 ).

\section{GAD-7 Anxiety Scale Scores}

A large portion of participants had symptoms of anxiety (167 [47.9\%]), with $28.9 \%$ of all participants in the mild range, $11.5 \%$ in the moderate range, and $7.4 \%$ in the severe range. For the question at the end of the GAD-7 asking "how difficult have these [symptoms] made it for you to do your work, take care of things at home, or get along with other people?," $48.1 \%$ of participants reported "somewhat difficult," $4.3 \%$ 
reported "very difficult," and $0.9 \%$ reported "extremely difficult." Females reported increased symptoms of anxiety $(\mathrm{p}=0.001)$ and increased difficulty with the getting work done, tasks at home, or getting along with other people $(\mathrm{p}=0.011)$ (Table 2A ). The median (IQR) score on the GAD-7 was 4.0 (2.0-8.0). Similar to findings in severity of symptoms, female participants had higher scores compared to males (6.0 [3.0-9.0] vs. 3.0 [1.8-7.0]; $\mathrm{p}<0.0005$ ) (Table 3A ). Multivariable logistic regression analysis also showed that females were more likely to report anxiety (OR 2.53, CI [1.59-4.02]; p<0.005) (Table 4 ).

\section{IES Distress Scale Scores}

A large portion of participants had symptoms of distress (210 [60.2\%]), with $32.7 \%$ of all participants in the mild range, $20.9 \%$ in the moderate range, and $6.6 \%$ in the severe range. Females reported experiencing higher symptoms of distress compared to males $(\mathrm{p}=0.001$, Table 2A $)$. Compared with those working in states with less than 20,000 positive cases, participants working in states with greater than 20,000 positive cases were significantly more likely to experience symptoms of distress on the IES $(p=0.027)$. Compared to those working in states with less than 1,000 deaths reported, participants working in states with greater than 1,000 reported deaths were also significantly more likely to experience symptoms of distress on the IES $(\mathrm{p}=0.013)($ Table 2B $)$.

The median (IQR) score on the IES for distress was 13.0 (3.0-27.0). The median (IQR) scores for the two sub-scores that comprise the distress score were 6.0 (2.0-13.0) for intrusive symptoms and 6.0 (1.0-14.0) for avoidance symptoms. Similar to findings in severity of symptoms, female participants had higher scores compared to males (19.0 [8.0-31.0] for females vs. 10.0 [2.0-22.2] for males; $\mathrm{p}<0.0005$;Table 3A ). Those who worked in a state with greater than 20,000 cumulative positive cases had significantly increased scores of distress compared to those who worked in a state with fewer than 20,000 positive cases (17.0 [5.0-29.0] vs. 10.0 [3.0-27.0]; $\mathrm{p}=0.021$ ), and this was also reflected in the two distress sub-scores (intrusive and avoidance). Working in a state with greater than 1,000 deaths had significantly increased scores of distress compared to working in a state with fewer than 1,000 deaths $(17.0[5.0-29.0]$ vs. 10.0 [2.0-27.0]; $\mathrm{p}=0.014)$, and this was also reflected in the two distress sub-scores. Lastly, geographic region was associated with significant differences in the intrusive sub-score of distress (Midwest 5.0 [1.0-13.0], Northwest 8.0 [3.0-15.0], South 3.0 [1.0-11.0], West 6.0 [1.0-13.0]; $\mathrm{p}=0.021$ ) (Table 3B ).

Multivariable logistic regression analysis also showed that females and physicians working in a state with greater than 20,000 positive cases were associated with increased presence of symptoms of distress (Table 4 ). Compared to males, females were more likely to report symptoms of distress (OR 2.68, CI [1.64-4.37]; $\mathrm{p}<0.0005)$. Compared to physicians working in a state where the number of positive COVID-19 cases was less than 20,000, those working in a state where the positive case number exceeded 20,000 were more likely to have symptoms of distress (OR 2.01, CI [1.22-3.31]; $\mathrm{p}=0.006$ ).

PHQ-2 Depression Scale Scores

$37(10.6 \%)$ participants were positive on our depression screener. In a clinical setting, patients who screen positive on this depression instrument would then require a more detailed screening to assess depression. The median (IQR) score on the PHQ-2 for depression for all participants was 0.0 (0.0-2.0). There were no significant differences in responses to the PHQ-2 between our subgroups.

\section{Discussion}

In this national survey-based study, we found large proportions of participants to be experiencing symptoms of burnout, anxiety, and distress. Furthermore, we identified demographic risk factors for presence of symptoms, including type of physician, sex, geographic region, and incidence of COVID-19. Turning attention to physician mental health is of great importance, as these problems have high prevalence in the physician population even prior to this pandemic. Studies conducted prior to the COVID-19 pandemic have reported prevalence of burnout in all physicians as greater than $50 \%,{ }^{34}$ and prevalence of depressive symptoms in surgeons as around $30 \% .{ }^{35}$ Though studies conflict regarding the exact prevalence of suicide in the US physician population, ${ }^{36}$ the suicide rate is widely cited as being higher than the general population. ${ }^{37}$ The 
increased pressures during the current pandemic have raised concerns for worsening mental health from this baseline ${ }^{38,39}$ Given the route of transmission of Sars-CoV-2, there is increased concern for otolaryngologists due to the multidisciplinary nature of the field and risk of aerosolization during procedures. ${ }^{4,6}$ To our knowledge, this is the only study that has specifically surveyed academic otolaryngologists during a pandemic using standardized metrics of burnout, anxiety, distress, and depression.

Our findings show that $47.9 \%$ of participants had symptoms of anxiety, $60.2 \%$ had symptoms of distress, $21.8 \%$ reported burnout, and $10.6 \%$ screened positive for depression symptoms. Out of the four, distress was the most prevalent positive result among our participants. Increased IES scores have correlated with a risk of PTSD, with Coffey et al. suggesting a cut-off of $27 .{ }^{31,40} 27.5 \%$ of our participants fall into the moderate and severe ranges, which start at a score of 26 . A score of 10 or greater on the GAD-7 is thought to be a reasonable cut-off point for identifying cases of generalized anxiety disorder, ${ }^{26}$ and $18.9 \%$ of our participants scored above that. The PHQ-2 assesses the degree to which an individual has experienced depressed mood and anhedonia over the past two weeks, serving as a screener for depression. It should be emphasized that a positive screening on PHQ-2 requires further evaluation with the PHQ-9 to make any conclusions, with $10.6 \%$ of our participants warranting additional screening. Overall, the reported symptoms in our study are concerning for the future mental wellbeing of our physicians, particularly regarding distress and anxiety, though further study is needed.

Residents reported increased burnout compared to attendings. This is unsurprising given the nature of their role in the hospital and the increased work hours. Prior studies have revealed significantly elevated levels of burnout in US otolaryngology residents compared to attendings at baseline. ${ }^{41}$ Importantly, these studies have used the Maslach Burnout Inventory (MBI) as their measure of burnout, which our Mini-Z burnout assessment has been validated against with particularly good correlation for the MBI's emotional exhaustion subscale. ${ }^{28}$ A study by Golub et al. reported high emotional exhaustion in $33 \%$ of residents, moderate in $29 \%$, and low in $38 \%$, which was strongly associated with increased work hours. ${ }^{42}$ In our study, $29.7 \%$ of residents reported at least "I am definitely burning out and have one or more symptoms of burnout, e.g., emotional exhaustion," and $70.3 \%$ of residents reported "I enjoy my work. I have no symptoms of burnout" or "I am under stress, and don't always have as much energy as I did, but I don't feel burned out." Taken together, it is possible that we are in fact seeing a decrease in burnout from baseline in our study. Though residents anecdotally report increased anxiety and stress in response to COVID-19, they also acknowledge that their work hours are much improved due to the cancellation of elective procedures and limitations on the number of in-hospital personnel. Thus, in the specific case of burnout, increased time off may have mitigated increased stressors in the workplace for our population.

Given the uneven spread of COVID-19 throughout the United States, we sought to identify an association between severity of COVID-19 and our mental health outcome measures. Our findings identified differences in distress based on these variables. Physicians working in states with greater than 20,000 positive cases or 1,000 deaths reported increased symptoms of distress compared to those in states with less than 20,00 positive cases or 1,000 deaths. When looking specifically at intrusive distress symptoms, separated out from the avoidant symptoms, there was a significant difference by region, with the Northeast having the highest median intrusive distress scores. As the Northeast had a substantially greater number of cases during our study period ${ }^{25}$ these participants are more likely to be treating positive patients or potentially being redeployed to other roles, and their stress may be compounded by diminishing PPE. Given the relationship between positive case numbers, death numbers, and region, only the positive case number variable was included in the multivariable analysis, and remained significant for distress.

Female respondents reported significantly higher amounts of burnout, anxiety, and distress. These findings are consistent with those identified in other studies during the current pandemic in China. ${ }^{21,22}$ This is also supported by an abundance of literature on a higher prevalence of "internalizing" psychiatric disorders such as anxiety and depression in females compared to males, who have higher prevalence of substance abuse and "externalizing" disorders, including attention-deficit/hyperactivity disorder, conduct disorder, intermittent explosive disorder, and oppositional defiant disorder. ${ }^{43,44}$ However, it is also important to consider the risks 
of response and measurement bias in these screening tools. These tools rely on symptom-based reporting, where males may be less likely to report symptoms. ${ }^{45-47}$ Furthermore, their symptoms may not fit these standard measurement tools, and their "externalizing" disorders may be masking depression and anxiety. ${ }^{46}$ For these reasons, it is possible that males may be underdiagnosed by these tools and clinically. Thus, our study may not be fully capturing the state of mental health among males, and therefore it is important to focus efforts on improving mental wellness in all physicians regardless of their gender.

This study has several limitations that are important to consider. Depending on the trajectory of the pandemic, the mental health symptoms of health care workers could intensify or diminish over time. Thus, long-term psychological implications of this population are worth future investigation. In addition, we did not include a control group and therefore are unable to definitely conclude that these symptoms in healthcare workers differ from those of the general population or of any other specialty. However, Zhang et al. found healthcare workers mental health scores to be significantly increased compared to nonmedical health care workers during COVID-19 in China. ${ }^{22}$ We are also unable to distinguish whether these symptoms are in the setting of preexisting mental health symptoms rather than new symptoms, though free responses to an optional question at the end of the survey suggest that many are experiencing a mental change that they attribute to COVID-19. Additionally, because our survey was emailed to each program director to distribute to their department, we are unable to confirm whether they received this email and/or forwarded it to their department. Given our response rates, we cannot exclude the possibility of a non-response bias. Providers who received but did not respond to the survey may not have been experiencing any distress, anxiety, burnout, or depression and therefore were not interested in responding. Alternatively, those who received the survey but did not fill it out could have been too overwhelmed to respond.

\section{Conclusion}

The COVID-19 pandemic presents a challenging position for otolaryngologists, where a calling to serve their communities may be at odds with concern for self and loved ones. Otolaryngologists are particularly at risk due to the aerosolizing nature of their procedures. We found that otolaryngologists are experiencing elevated anxiety and distress. However, the cancellation of elective procedures and measures to limit in-house personnel may also have provided an increase in time outside of work, mitigating some of the negative effects of the pandemic on mental health. We present here a cross-sectional analysis of a spectrum of mental health concerns among otolaryngologists' during this pandemic. Though further studies are needed to capture a longitudinal picture, it is important that institutions start focusing efforts on the mental wellness of their physicians.

\section{References}

1. Givi B, Schiff BA, Chinn SB, et al. Safety Recommendations for Evaluation and Surgery of the Head and Neck During the COVID-19 Pandemic. JAMA Otolaryngology-Head \& Neck Surgery. Published online March 31, 2020. doi: doi:10.1001/jamaoto.2020.0780

2. Vargas M, Servillo G. Improving staff safety during tracheostomy in COVID-19 patients. Authorea . March 31, 2020. doi: https://doi.org/10.22541/au.158567936.68111075

3. Rassekh CH, Jenks CM, Ochroch EA, Douglas JE, O'Malley Jr. BW, Weinstein GS. Management of the Difficult Airway in the COVID-19 Pandemic: An Illustrative Complex Head and Neck Case Scenario.Authorea . April 02, 2020. doi: https://doi.org/10.22541/au.158585426.67421372

4. Tran K, Cimon K, Severn M, Pessoa-Silva CL, Conly J. Aerosol Generating Procedures and Risk of Transmission of Acute Respiratory Infections to Healthcare Workers: A Systematic Review. PLoS ONE . 2012;7 (4):e35797. doi: https://dx.doi.org/10.1371\%2Fjournal.pone.0035797

5. Europe's Doctors Repeat Errors Made in Wuhan, China Medics Say. Bloomberg News. Published March 17, 2020. Accessed April 5, 2020. https://www.bloomberg.com/news/articles/2020-03-17/europe-s-doctorsgetting-sick-like-in-wuhan-chinese-doctors-say 
6. Workman AD, Welling DB, Carter BS, et al. Endonasal instrumentation and aerosolization risk in the era of COVID-19: simulation, literature review, and proposed mitigation strategies. International Forum of Allergy $E$ S Rhinology . 2020. doi: https://doi.org/10.1002/alr.22577

7. Kowalski LP. COVID-19 pandemic: effects and evidence-based recommendations for otolaryngology and head and neck surgery practice.Authorea . March 31, $2020 . \quad$ doi: https://doi.org/10.22541/au.158567937.74092406

8. Bann DV, Patel VA, Saadi R, et al. Impact of Coronavirus (COVID-19) on Otolaryngologic Surgery: A Brief Commentary. Authorea. March 31, 2020. doi: https://doi.org/10.22541/au.158567936.61156499

9. Husain I. Why COVID-19 Is a Special Danger to Otolaryngologists. Scientific American. April 24, 2020. Accessed May 6, 2020. https://blogs.scientificamerican.com/observations/why-covid-19-is-a-special-dangerto-otolaryngologists/

10. Livingston E, Desai A, Berkwits M. Sourcing Personal Protective Equipment During the COVID-19 Pandemic. JAMA . 2020. doi: doi:10.1001/jama.2020.5317

11. Maunder R, Hunter J, Vincent L, et al. The immediate psychological and occupational impact of the 2003 SARS outbreak in a teaching hospital. CMAJ . 2003;168 (10):1245-51.

12. Bai Y, Lin C-C, Lin C-Y, Chen J-Y, Chue C-M, Chou P. Survey of Stress Reactions Among Health Care Workers Involved With the SARS Outbreak. Psychiatric Services . 2004;55 (9):1055-57. doi: 10.1176/appi.ps.55.9.1055

13. Chua SE, Cheung V, Cheung C, et al. Psychological Effects of the SARS Outbreak in Hong Kong on High-Risk Health Care Workers. The Canadian Journal of Psychiatry . 2004;49 (6):391-93. doi: $10.1177 / 070674370404900609$

14. Verma S, Mythily S, Chan YH, Deslypere JP, Teo EK, Chong SA. Post-SARS psychological morbidity and stigma among general practitioners and traditional Chinese medicine practitioners in Singapore. Ann Acad Med Singapore . 2004;33 (6):743-8.

15. Lehmann M, Bruenahl CA, Addo MM, et al. Acute Ebola virus disease patient treatment and healthrelated quality of life in health care professionals: A controlled study. Journal of Psychosomatic Research . 2016;83:69-74. doi: 10.1016/j.jpsychores.2015.09.002

16. Gershon R, Dernehl LA, Nwankwo E, Zhi Q, Qureshi K. Experiences and Psychosocial Impact of West Africa Ebola Deployment on US Health Care Volunteers. PLoS Curr . 2016;8 . doi: doi:10.1371/currents.outbreaks.c7afaae124e35d2da39ee7e07291b6b5

17. Ji D, Ji Y-J, Duan X-Z, et al. Prevalence of psychological symptoms among Ebola survivors and healthcare workers during the 2014-2015 Ebola outbreak in Sierra Leone: a cross-sectional study. Oncotarget . 2017;8 (8). doi: 10.18632/oncotarget.14498

18. Lee AM, Wong JG, McAlonan GM, et al. Stress and Psychological Distress among SARS Survivors 1 Year after the Outbreak. The Canadian Journal of Psychiatry . 2007;52 (4):233-40. doi: 10.1177/070674370705200405

19. Liu X, Kakade M, Fuller CJ, et al. Depression after exposure to stressful events: lessons learned from the severe acute respiratory syndrome epidemic. Comprehensive Psychiatry . 2012;53 (1):15-23. doi: 10.1016/j.comppsych.2011.02.003

20. Lung F-W, Lu Y-C, Chang Y-Y, Shu B-C. Mental Symptoms in Different Health Professionals During the SARS Attack: A Follow-up Study.Psychiatric Quarterly . 2009;80 (2):107-16. doi: 10.1007/s11126-0099095-5

21. Lai J, Ma S, Wang Y, et al. Factors Associated With Mental Health Outcomes Among Health Care 
Workers Exposed to Coronavirus Disease 2019.JAMA Network Open . 2020;3 (3):e203976. doi: 10.1001/jamanetworkopen.2020.3976

22. Zhang W-R, Wang K, Yin L, et al. Mental Health and Psychosocial Problems of Medical Health Workers during the COVID-19 Epidemic in China. Psychotherapy and Psychosomatics . 2020:1-9. doi: $10.1159 / 000507639$

23. Chew NWS, Lee GKH, Tan BYQ, et al. A multinational, multicentre study on the psychological outcomes and associated physical symptoms amongst healthcare workers during COVID-19 outbreak. Brain, Behavior, and Immunity . 2020. doi: 10.1016/j.bbi.2020.04.049

24. Institute for Health Metrics and Evaluation (IHME). COVID-19 Projections. Seattle, WA: IHME, University of Washington. 2020. Accessed April 26, 2020. https://covid19.healthdata.org/projections

25. The COVID Tracking Project. 2020. Accessed April 26, 2020. https://covidtracking.com/

26. Spitzer RL, Kroenke K, Williams JBW, Löwe B. A Brief Measure for Assessing Generalized Anxiety Disorder. Archives of Internal Medicine . 2006;166 (10):1092. doi: 10.1001/archinte.166.10.1092

27. Dolan ED, Mohr D, Lempa M, et al. Using a Single Item to Measure Burnout in Primary Care Staff: A Psychometric Evaluation. Journal of General Internal Medicine . 2015;30 (5):582-87. doi: 10.1007/s11606014-3112-6

28. Rohland BM, Kruse GR, Rohrer JE. Validation of a single-item measure of burnout against the Maslach Burnout Inventory among physicians.Stress and Health . 2004;20 (2):75-79. doi: 10.1002/smi.1002

29. Horowitz M, Wilner N, Alvarez W. Impact of Event Scale: A Measure of Subjective Stress. Psychosomatic Medicine . 1979;41 (3):209-18. doi: 10.1097/00006842-197905000-00004

30. Kroenke K, Spitzer RL, Williams JBW. The Patient Health Questionnaire-2. Medical Care . 2003;41 (11):1284-92. doi: 10.1097/01.mlr.0000093487.78664.3c

31. Coffey SF, Gudmundsdottir B, Beck JG, Palyo SA, Miller L. Screening for PTSD in motor vehicle accident survivors using the PSS-SR and IES. 2006;19 (1):119-28. doi: 10.1002/jts.20106

32. Vickery TW, Weterings R, Cabrera-Muffly C. Geographic Distribution of Otolaryngologists in the United States. Ear, Nose 63 Throat Journal . 2016;95 (6):218-23. doi: 10.1177/014556131609500607

33. Table B3. Number of Active Residents, by Type of Medical School, GME Specialty, and Sex. AAMC. Sept 5, 2019. Accessed May 3, 2020. https://www.aamc.org/data-reports/students-residents/interactivedata/report-residents/2019/table-b3-number-active-residents-type-medical-school-gme-specialty-and-sex

34. Shanafelt T, Hasan O, Dyrbye L, et al. Changes in Burnout and Satisfaction With Work-Life Balance in Physicians and the General US Working Population Between 2011 and 2014. Mayo Clin Proc . 2015;90 (12):1600-13. doi: 10.1016/j.mayocp.2015.08.023.

35. Shanafelt T, Balch C, Bechamps G, et al. Burnout and Career Satisfaction Among American Surgeons. Annals of Surgery . 2009;250 (3):463-71. doi: 10.1097/SLA.0b013e3181ac4dfd

36. Eckleberry-Hunt J, Lick D. Physician Depression and Suicide: A Shared Responsibility. Teach Learn Med . 2015;27 (3):341-5. doi: 10.1080/10401334.2015.1044751.

37. Decaporale-Ryan L, Sakran JV, Grant SB, et al. The undiagnosed pandemic: Burnout and depression within the surgical community.Current Problems in Surgery . 2017;54 (9):453-502. doi: 10.1067/j.cpsurg.2017.07.001

38. Holmes EA, O'Connor RC, Perry VH, et al. Multidisciplinary research priorities for the COVID-19 pandemic: a call for action for mental health science. The Lancet Psychiatry . 2020. doi: 10.1016/s22150366(20)30168-1 
39. Shanafelt T, Ripp J, Trockel M. Understanding and Addressing Sources of Anxiety Among Health Care Professionals During the COVID-19 Pandemic. JAMA . 2020. doi: 10.1001/jama.2020.5893

40. Wohlfarth T, van den Brink W, Winkel F, ter Smitten M. Screening for Posttraumatic Stress Disorder: an evaluation of two self-report scales among crime victims. Psychol Assess . 2003;15 (1):101-9. doi: 10.1037/1040-3590.15.1.101

41. Pulcrano M, Evans SRT, Sosin M. Quality of Life and Burnout Rates Across Surgical Specialties: A Systematic Review. JAMA Surgery . 2016;151 (10):970. doi: 10.1001/jamasurg.2016.1647

42. Golub JS, Weiss PS, Ramesh AK, Ossoff RH, Johns MM. Burnout in Residents of Otolaryngology-Head and Neck Surgery: A National Inquiry into the Health of Residency Training. Academic Medicine . 2007;82 (6):596-601. doi: 10.1097/acm.0b013e3180556825

43. Seedat S, Scott KM, Angermeyer MC, et al. Cross-National Associations Between Gender and Mental Disorders in the World Health Organization World Mental Health Surveys. Archives of General Psychiatry . 2009;66 (7):785. doi: 10.1001/archgenpsychiatry.2009.36

44. Eaton NR, Keyes KM, Krueger RF, et al. An invariant dimensional liability model of gender differences in mental disorder prevalence: Evidence from a national sample. Journal of Abnormal Psychology . 2012;121

(1):282-88. doi: $10.1037 / \mathrm{a} 0024780$

45. Courtenay WH. Constructions of masculinity and their influence on men's well-being: a theory of gender and health. Social Science \& Medicine . 2000;50 (10):1385-401. doi: 10.1016/s0277-9536(99)00390-1

46. Smith DT, Mouzon DM, Elliott M. Reviewing the Assumptions About Men's Mental Health: An Exploration of the Gender Binary. American Journal of Men's Health . 2018;12 (1):78-89. doi: $10.1177 / 1557988316630953$

47. Martin LA, Neighbors HW, Griffith DM. The Experience of Symptoms of Depression in Men vs Women. JAMA Psychiatry . 2013;70 (10):1100. doi: 10.1001/jamapsychiatry.2013.1985

\section{Hosted file}

Table_1 (1).docx available at https://authorea.com/users/312328/articles/450601-mentalhealth-among-otolaryngology-resident-and-attending-physicians-during-the-covid-19pandemic-a-national-study

\section{Hosted file}

Table_2A.docx available at https://authorea.com/users/312328/articles/450601-mental-healthamong-otolaryngology-resident-and-attending-physicians-during-the-covid-19-pandemic-anational-study

\section{Hosted file}

Table_2B.docx available at https://authorea.com/users/312328/articles/450601-mental-healthamong-otolaryngology-resident-and-attending-physicians-during-the-covid-19-pandemic-anational-study

\section{Hosted file}

Table_3A.docx available at https://authorea.com/users/312328/articles/450601-mental-healthamong-otolaryngology-resident-and-attending-physicians-during-the-covid-19-pandemic-anational-study

\section{Hosted file}


Table_3B.docx available at https://authorea.com/users/312328/articles/450601-mental-healthamong-otolaryngology-resident-and-attending-physicians-during-the-covid-19-pandemic-anational-study

\section{Hosted file}

Table_4 (1).docx available at https://authorea.com/users/312328/articles/450601-mentalhealth-among-otolaryngology-resident-and-attending-physicians-during-the-covid-19pandemic-a-national-study 